\title{
Library Computer Database Management Application
}

\author{
Yingli Wang \\ Guangzhou Huali Science and Technology Vocational College, \\ Guangzhou 511325, China. \\ 105624725@qq.com.
}

Keywords: Library; Computer database; Management application

\begin{abstract}
The library is of great significance to the development of education in our country. Its management cannot be ignored. In the management of the library, the library computer database management is currently the most common and practical method. The author conducts research and analysis on the application of library computer database management in our country. The application of library computer database management has important advantages in library management, and it is an indispensable technical method for library management in China. The article starts with the management of library application computer database, and discusses the application of computer database management technology to the characteristics of computer database.
\end{abstract}

\section{Introduction}

With the continuous development of computer technology, the computer database system has become an effective organizational system for the implementation of database applications, and it has been widely applied to various fields, which has a very good role in promoting the development of China's information. As the largest library of book information storage, the introduction of computer data management system is a necessary choice. Therefore, strengthening the maintenance of database security and establishing a sound library computer management system will greatly promote library computer management.

\section{The characteristics of library computer database management}

\subsection{Completeness}

The database is a very important part of the computer. It is a very important part of the computer and guarantees the security and reliability of the computer. Therefore, it must be complete for the library computer database. The integrity of the database contains a comparison. More content mainly includes the validity, consistency and correctness of database data.

\subsection{Figure independence}

The library computer database is a very important part of the library management. The independence is a very important feature for the library computer database. The independence referred to here mainly includes two aspects. First, physical independence means that the database is stored in the computer's physical location relative to the other application software; the second is the logical independence, referring to the database. Logically separate from other applications on the computer.

\subsection{Security}

The library computer database stores a large amount of information and its security cannot be ignored. The security here refers mainly to the security of the information stored in the database, to the security of the database, on the contrary, the information is stolen, falsified and lost.

\subsection{Concurrency control}

In the library, book sharing is a very important feature of the library. Therefore, the library computer database is required to have the ability of concurrency control to achieve the purpose of resource sharing. In this way, users can focus on the retrieval and reading of book resources through a unified application program, and prevent the occurrence of data inconsistency. 


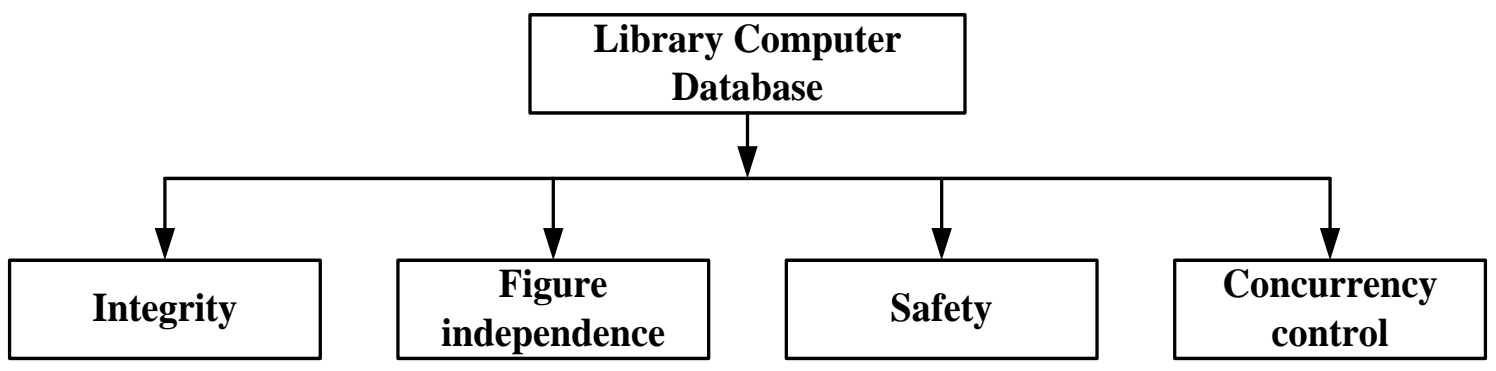

Figure 1 The Characteristics of Library Computer Database Management.

\section{3. the application of library computer database}

\subsection{The literature is too fragmented and the management system is not uniform.}

The number of books in major libraries can be described in countless numbers. There are only books we haven't seen before and there are no books we can't find. It is precisely because of too many kinds of species and the large number of books that have been collected. This has led to a variety of literature and material distributions. In addition to the lack of a complete resource management system, it has taken a lot of trouble to find the books that they need. Therefore, this is an urgent need for us to solve them [1] .

\subsection{Different library database application systems vary.}

With the rise of digital libraries, more and more libraries have purchased computers, conducted related data management, and used computerized data management. This is more effective than traditional manual management and improves the efficiency of management, but due to the various libraries The systems used in the database are different. Users who want to go to different libraries must first familiarize themselves with their respective management systems before they can search for relevant books and materials [2] . The inconsistent database management system creates many unnecessary problems for the users. It also increases the workload of the library. Therefore, it is very important to establish a unified database management platform.

\section{The application analysis of library computer database}

\subsection{Database Management System Technical Analysis}

Access management technology. Access management technologies include access technology applications and user authentication technologies. Access control technology refers to restricting the rights and scope of the user's use of resources based on the user's identity information. The application of this technology can not only restrict users' access to unauthorized resources, but also prevent illegal users from invading protected resources [3] .

Apply encryption technology. Encrypting the computer storage database is an important means to ensure the security of document data. At present, there are two types of encryption methods for library databases: symmetric key algorithms and public key algorithms. The use of database encryption technology can not only prevent unauthorized users from unauthorized access, but also effectively avoid data theft. It is of great significance to the protection of data security, and can also effectively improve the applicable performance of the database [4] .

Data backup and recovery technologies. Data backup and recovery are prevention and remedial measures to prevent the loss or destruction of book information. As we all know, the database system is established on the basis of the computer, due to the computer power failure, software failure and system failure, will stop the operation of the database, so that data is destroyed or lost. Therefore, it is necessary to backup the data in advance. Currently, there are three common backup methods: dynamic backup, static backup, and logical backup. There are also three common recovery technologies: database backup, disk mirroring, and online logging [5] . 


\subsection{To achieve resource sharing, and increase the auxiliary processing functions}

An important management object in the library computer library management system is the literature data, which is independent of the application software and needs to be stored separately. In the process of managing various business operations of the library, it is necessary to repeatedly aggregate and process bibliographic data information to form a large number of single, capable of reflecting and revealing the contents and forming features of the documents. For example, a book or the author and title of a document needs to be reflected in the optional card for the interviewing business, in the property book of the registered business, and in the various items described in the cataloging business. However, the same literature and data information repeatedly appear between different business management departments in the library, which causes the main reason for the low efficiency of the library. If the computer database is used to control and manage the various business operations in the library, the occurrence of the above situations can be effectively avoided, and it can largely achieve the purpose of one-time input and multiple-time output of the document data, making the document Data information can be fully shared in the library. Data sharing requires that the structure of each document data stored in the computer processing system in the library should have a unified and reasonable standard, and requires the document data listed in each record to be independent, share high, and have many attributes. And so on, the system can make the information of the stored document data more accurate and detailed. Furthermore, by observing the use of the literature, it can be found that the degree of aging and the rate of aging of the various disciplines are different. Therefore, the literature data in the library computer database should be dynamically combined. In order to reduce the increasing redundant literature information in the system to a great extent, and to further promote the security and accuracy of the system literature data, the computer database should also have the modification, reality, query, and deletion of document resources. The modern auxiliary processing capabilities, which can make a fundamental change in the library's work and service levels, and the library's computer database can fully achieve the higher levels of on-line cataloging, on-line procurement, online retrieval, etc. Resource Sharing.

\subsection{Realize the substitution and expansion of traditional libraries}

Alternatives to traditional libraries. The substitution of traditional libraries refers to the substitution of the contents of all the manual business management work in the library, including the collection and sorting of the library's documents, and also the work of services such as the purchase of books. The actual work of registration, cataloging, circulation, etc., as well as some hard-to-finish work by hand, can all be achieved using computer databases, thus facilitating the digitization and automated control and management of libraries. The application of the computer database makes the library get rid of the limitations of the traditional document processing, making the library use the computer database to establish an open service system in terms of collection, processing, and service of document information, making the library not only It is limited to the service of users in the region and it can also serve users in other regions.

The expansion of traditional libraries. The expansion of the library refers to the adoption of a computer database, thereby replacing most of the library's business management efforts, while improving the functions of the library and further deepening and strengthening it. Due to the limitation of time and space, the traditional library mainly uses the library as the main library, and its service scope is also limited to the library. The library has been affected by digitization and caber information. Today's libraries have begun to develop towards openness and socialization in an all-round way, achieving the greatest degree of resource sharing, especially the application of computer databases, making users of the literature. Access, retrieval, and lending are no longer confined to the library, allowing the sharing of documentary information resources to the greatest degree, completely breaking down the restrictions of the regions and institutions, and relegating their own digital resources to a large library. The components of the system. In order to better satisfy the readers' needs, the library's information service channels should be continuously strengthened, such as document delivery, inter library loan, and so on. 


\subsection{Library Database Design}

According to the needs of users, scientific and technical personnel produce a conceptual model of user's viewpoints, and then turn these conceptual patterns into theoretical logic patterns, so that this user concept pattern is independent and the complex patterns are transformed into relatively single patterns, which can reduce the number of users. Other system limitations. The establishment of a library database can be roughly divided into three aspects. First, design a local ER. Second, integrate these local ERs to make it a global model. Finally, optimize the global model. The so-called logical model is the process of transforming the ER diagram into a relational model, which ultimately completes the database design of the library.

\subsection{How computers operate in the integration of library resources}

Computer application technology can be summarized in the following terms in library management: circulation, storage, editing, management of periodicals, updating, search, and so on. We can manage the literature through the computer's own software system. The main ones include the above functions. We can use the computer system to conduct a unified purchase of the library's literature resources, make a unified catalog, and conduct a variety of periodicals and magazines. Orderly arrangement, establish a unified database platform. Through computer technology, users can reduce the time spent on the search and quickly find the location of the book. The department with the greatest work intensity in the library is the circulation department, because the original borrowing program is complicated and delays a lot of time. The dissatisfaction of many readers, however, through the application of computer technology, has greatly eased the work burden of the circulation department, and the corresponding work can be accomplished by just pressing a few keyboards, which not only facilitates the user but also reduces the staff of the library. Workload.

There are three kinds of computer operating modes: microcomputer single-user stand-alone system, minicomputer muti-user system and computer local network system. In the library, it is impossible to use the first two systems. The first two systems have many shortcomings and cannot meet the needs of the library. Therefore, the library uses a local computer network system. The local network system of the microcomputer can better serve the library and can achieve resource sharing, an efficient file service system, and operational reliability. This mode of operation has been trusted by a large number of users and has been recognized by many large libraries. Therefore, in the future development trend, there will be more libraries using microcomputer local network systems.

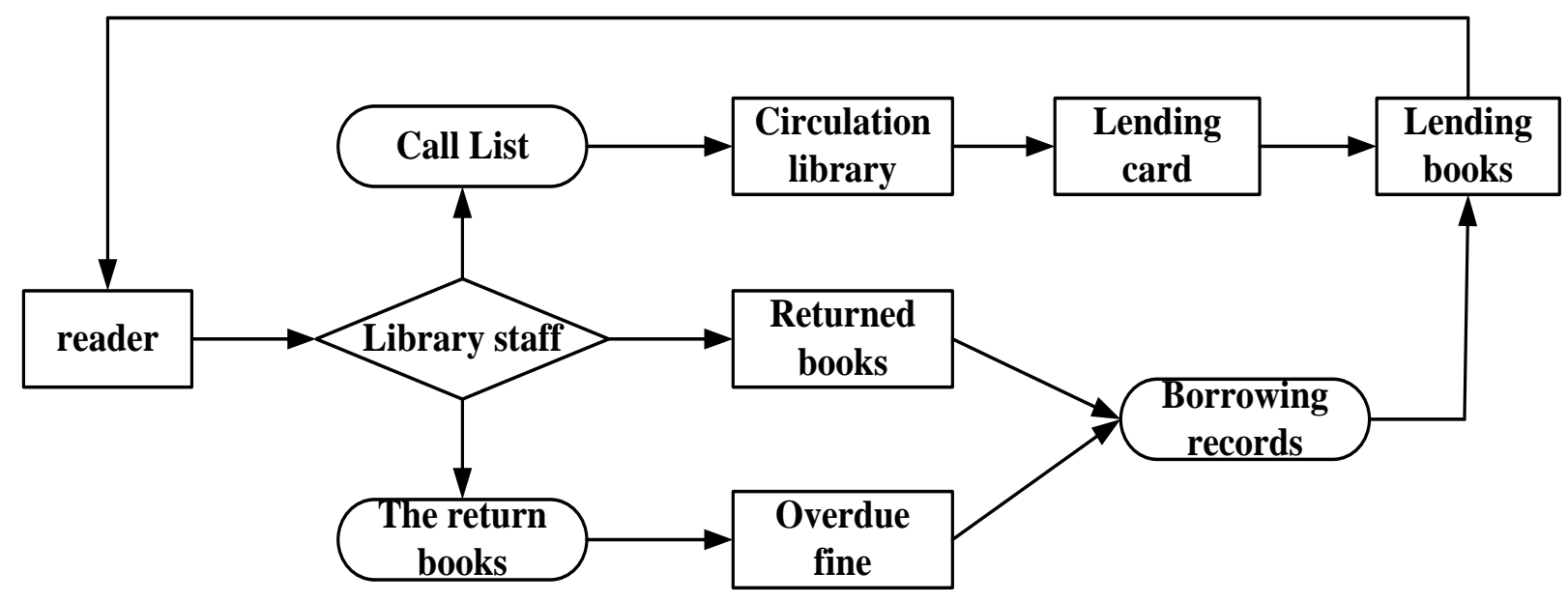

Figure 2 Book business flow chart.

\section{The library computer database management measures}

\subsection{Strengthen the security maintenance of computer network systems}

The maintenance of the computer network system should be based on a comprehensive understanding of the network components of each part of the library, and then a detailed line inspection system and network switching equipment should be developed, and the client should be 
regularly maintained except In addition to this, it is also necessary to make full use of firewalls or routers to manage the computer network more effectively. The configuration of the router enables the various departments of the library to have different usage rights for the network. In the process of enhancing the security maintenance of computer network systems, the most important point is the prevention of computer viruses. Computer viruses are compiled and manufactured by humans, and they can destroy computer systems and steal information. To a large extent, it affects the computer's operating procedures. Therefore, it should pay attention to the installation of anti-virus software on computers in libraries, and it should also be constantly updated. The anti-virus software installed in the library can also be combined with a firewall so that the virus can be completely isolated from the internal network. Once a virus is found, it should be processed immediately to prevent the spread of the virus and affect the daily work of the library. .

\subsection{Improve the security of data in libraries}

The staff of the library should pay attention to the regular backup of the library's computer database. The data backup can not only ensure the security of the library computer database to a large extent, but also enable parallel operation of the data in the library. The applicable performance of the database is greatly improved, and it can also be implemented in the case where the user does not shut down the computer and replace the failed part. The encryption of library computer database mainly includes two methods, namely symmetric key algorithm and public key algorithm.

\subsection{Establish a Sound Safety Management System}

The security management system of library computer database includes the responsibility system of managers and operators, the daily data backup and recovery system of users, the daily management system of the computer main room in the library, various types of password systems, and the prevention of computer viruses. Monitoring system and so on. Only under the premise of establishing a sound safety management system can we achieve the maximum standardization of the library computer database management system, and can also improve the accountability of managers, and thus ensure the safe operation of the computer database in the library., And can make the staff in the library more efficient to complete the library's security management. Therefore, only when the enforcement of the system is strengthened can the normative behavior be carried out and the standards be followed, so that the various types of staff in the library can perform their duties and the security measures in the library. And the management system is integrated.

\section{Library Management System Maintenance}

According to the life cycle of system development, system maintenance belongs to the fifth stage. Broadly speaking, system maintenance involves the entire life cycle, including application system maintenance, data maintenance, code maintenance, document maintenance, and hardware device maintenance. Due to the use of structured module design, system maintenance costs and workload are relatively small: when a module needs to be modified or a module is added, it is only necessary to change or add this module, but it will not produce larger ones for other modules. Influencing; At the same time, the use of structured program design methods to standardize the interaction between the module structure and modules, and improve the maintainability of the system. After a period of commissioning and operation, the system adopts measures such as correctness maintenance, adaptive maintenance, perfecta maintenance and preventive maintenance according to different situations, and has performed many errors or functional deficiencies or changes in system development and operation. Repeated maintenance has ensured the safe operation of the system and basically achieved the planned goals of system development.

A development method that uses a combination of structured system development methods, life cycle methods, and prototype methods. Firstly, the life cycle method is used to determine the system development cycle and steps, and then the structured prototype development method is used to "structure" the initial prototype obtained using the prototype method, and the prototype is continuously modified and perfected during the development process. Powerful query function, flexible statistical strategy, and decision support. The search method is flexible, supports fuzzy queries and multiple nested combination queries; the statistical approach is extremely rich, and users 
can freely define, arbitrarily select, and freely arrange retrieval points; and the statistics are rich in content, and can be used to count readers' situations, collections, and distribution of books and periodicals. Circulation and lending provide a comprehensive reference for library business monitoring and decision making.

User-friendly interface, convenient and flexible operation. The system makes full use of advanced interface means, provides a large number of preset template definitions, built-in system online help, clear information tips, effectively reducing the difficulty of the use of the software, and easy operation. Complete rights control, data security. The system uses identity authentication. Only users with the appropriate permissions can perform corresponding operations. Otherwise, they cannot log in or operate on data. The system uses a web server and a main server to be separated. The firewall is installed on the server and the main library server is used. External isolation ensures that the system will not be attacked from the outside by using real-time or regular backups to ensure the security and integrity of the data. The system configuration is flexible and is suitable for the needs of different museums and policies. Comprehensive consideration of the actual situation of different levels of university libraries, for different library needs and policy differences, the system provides flexible parameter settings, users can build a business environment with the characteristics of the museum to meet the actual situation of the museum's conditions and policies need. Strict standardization and open interfaces enable resource sharing. The system adheres to strict prevailing standards and agreements, which are conducive to joint procurement, joint cataloging, inter library loan and resource sharing. Flexible printing. Built-in print preview and parameter setting generator, the user can output the print format. Database design specifications. The system uses an advanced development platform to design the database according to the database normalization theory to ensure the integrity and consistency of the data and ease of use, and to facilitate data maintenance.

\section{Conclusion}

With the rapid development of scientific research such as computer technology and database theory, the computer database is also developing in the process of maturing, and the application of computer database in the library has gradually gained popularity. Therefore, we should continue to explore the application of computer databases in libraries, and we should continue to strengthen the safety management of computer databases in libraries based on our understanding of their applications, so that computer databases can be maximized in libraries. The efficiency of various tasks.

\section{References}

[1]. Autumn Yu, et al. Analysis of the application of computer database management system . Silicon Valley. Vol.1, (2013)No. 10, p. 141-142.

[2]. Zhang Jin bracelet, et al. Library Computer Network System Maintenance and Management . Modern Library and Information Technology. Vol.3(2010)No. 11, p. 23-27.

[3]. Lin Jia, et al. Reflections on the Construction of Library Union under the Network Environment . Journal of Library Science in China. Vol.2 (2013)No. 6, p.115-118.

[4]. Li Gang, et al. On the Library Computer Management System. Vol. Journal of Zhejiang Shuren University. (2012)No. 12, p.251-254.

[5]. Song Ting, et al. Library computer database management application parsing.enterprise Herald. Vol.3 (2015) No. 46, p.356-359. 